\title{
INVESTIGATION ON SEPARATION EFFICIENCY OF LIQUID/SOLID HYDROCYCLONE ${ }^{*}$
}

\author{
WANG Li-yang, ZHENG Zhi-chu, GUO Jun, ZHANG Jun, TANG Chi \\ Institute of Mechanics, Chinese Academy of Sciences, Beijing 100080, China \\ E-mail : wangliyang@imech.ac.cn
}

\begin{abstract}
Liquid/solid hydrocyclones are widely used for various applications. Based on massive experiments, the relationships between separation efficiency and liquid velocity, oil/water ratio, as well as the number of hydrocyclones were investigated. The phenomenon of smaller particles more sensitive to changes of parameters was explained theoretically through force balance analysis. Besides, the dependence of separation efficiency on the Reynolds number and the Stokes number was regressed based on dimensional analysis. Finally, some valuable suggestions were put forward for the practical application of liquid/solid hydrocyclones.
\end{abstract}

KEY WORDS: hydrocyclone; liquid/solid separation; separation efficiency; dimensional analysis

\section{Introduction}

During the daily operation of many oil fields throughout the world, sand particles are required to be separated from liquid mixture because they could cause many troubles, including erosion, solids accumulation, and even equipment blockage, etc. Therefore, desanding facilities must be developed for the purpose of removing sand as much as possible before the liquid mixture enters into gathering/ transportation system.

Compared to more conventional solutions, such as filtration and gravitational settling, liquid/solid hydrocyclone offers several advantages, such as compact dimensions, operational simplicity, high separation efficiency and an ability to handle oily solids without causing blockage. Thus this technology has been used widely in many field applications. For example, Cyclotech initiated a Joint Industry Project supported by BP, Chevron, LASMO, Shell International and Statoil, and the work led Cyclotech to develop a type of wellhead desanding hydrocyclone (shown in Fig. 1) to Expro North Sea for use on the Shell Brent Delta platform, allowing problematic solids to be removed from the wellhead liquid mixture upstream.

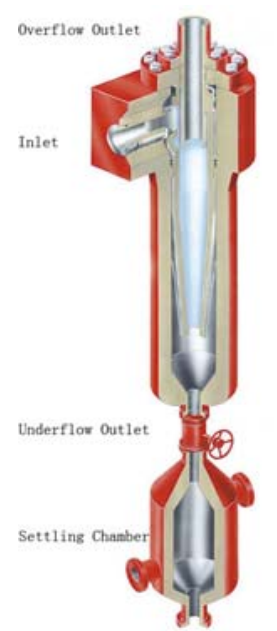

Fig. 1 Wellhead desanding cyclone

Recently, more and more attention has been paid to the development of offshore oil and gas fields due to the urgent need for energy. To solve many scientific and technological problems encountered, some key research subjects have been conducted jointly by Chinese Academy of Sciences and China National Offshore Oil Cooperation during the tenth "five-year" period. As one of the subjects, the research work of removing solid particles from oil/water mixture was undertaken by the Institute of Mechanics, CAS. A new set of experimental facilities given in Fig. 2 has been established in order to simulate the separation process of oil/water/sand particles in laboratory, of which the initial object is that separation efficiency of sand particles less than 80 eyes can arrive at 95 percent.

The schematic diagram of liquid/solid separation system is shown in Fig. 3. As can be seen, the system mainly consists of three parts: (I) feeding module,

\footnotetext{
* Project supported by the China National Offshore Oil Cooperation
} 


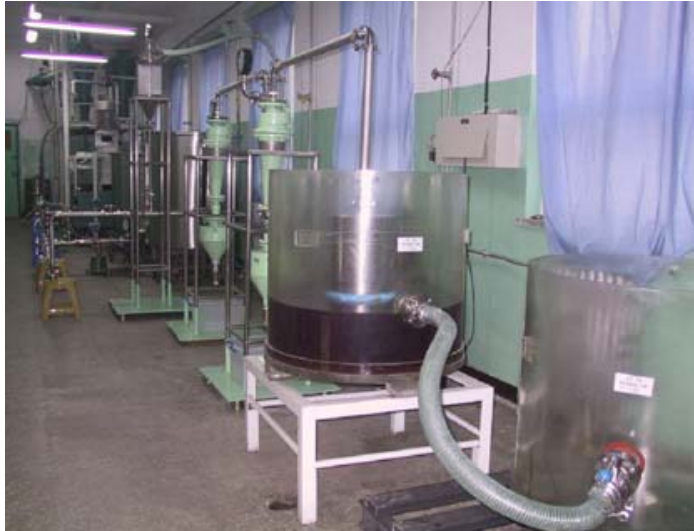

Fig. 2 Photo of liquid/solid separation system

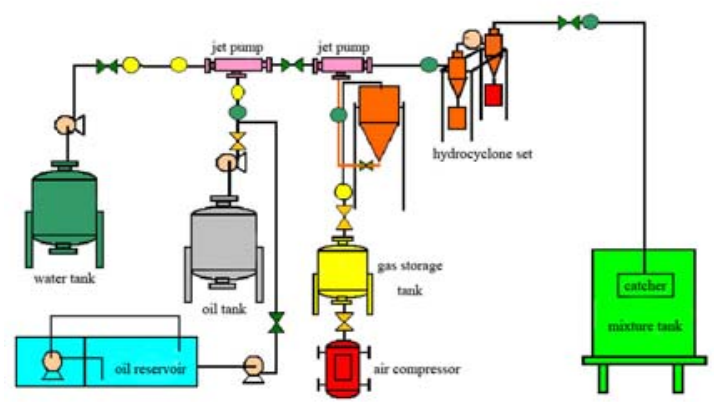

Fig. 3 Schematic diagram of separation system

including gas inlet pipe, water inlet pipe, sand-particle feeding pipe, pressure gauge and valves, etc. (II) the first hydrocyclone and the second one, which are the core components of the experimental system (III) catcher, including filtering screen and an overflow cylinder.

\section{Experiment}

\subsection{Experimental sytem}

During the experiments, water and oil were metered and supplied separately from two storage tanks. After having been fully mixed with oil/water mixture from jet pump, the sand particles fed into the hopper were introduced into hydrocyclones, where part of them separated would be collected at the corresponding settling chamber, while those fine particles escaped from the overflow outlet of the second hydrocyclone would be collected inside the catcher.

The fluids used in the present work were LP-14 white oil and tap water, and the viscosity-temperature curve of the former is shown in Fig. 4. The range of experimental parameters is as follows: oil/water mixture velocity $0-3.0 \mathrm{~m} / \mathrm{s}$, water cut $0.45-1.0$, and the average solid concentration at the inlet of the first hydrocyclone $8-18 \mathrm{~kg} / \mathrm{m}^{3}$.

\subsection{Experimental results}

Separation efficiency is of great significance in evaluating the performance of a liquid/solid hydro- cyclone, mainly depending on particle diameter, inlet velocity and liquid composition, which are consequently altered for each run to examine their effects on separation efficiency. Besides, due to the fact that in field applications the efficiency is often improved by installing the hydrocyclones in series, its influence on separation efficiency is also investigated.

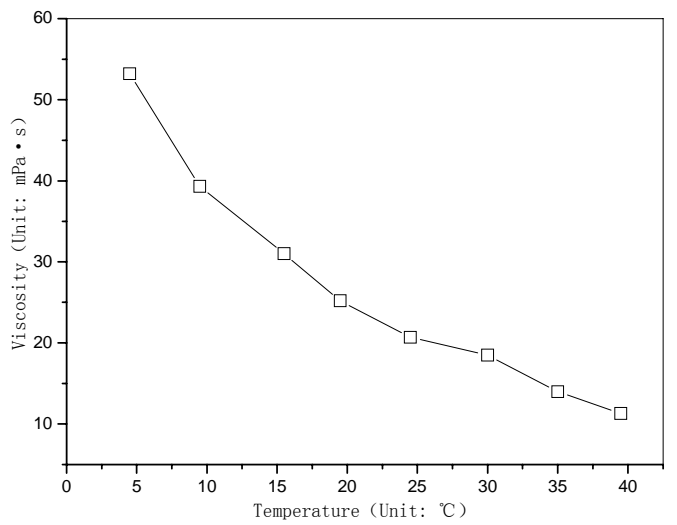

Fig. 4 Viscosity-temperature curve of LP-14 white oil

\subsubsection{Inlet velocity}

Fig. 5 compares the grade efficiency of the first hydrocyclone as the inlet velocity increases from $1.2 \mathrm{~m} / \mathrm{s}, 1.5 \mathrm{~m} / \mathrm{s}$ to $1.7 \mathrm{~m} / \mathrm{s}$ for a given feed size distribution and a constant water cut of 0.45 .

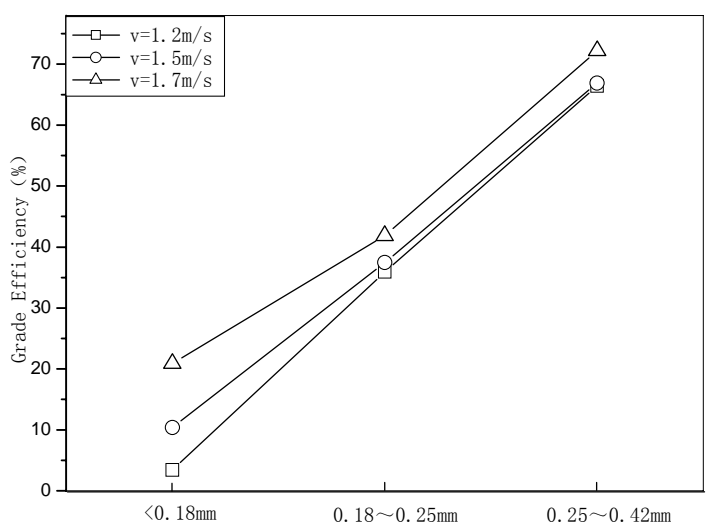

Fig. 5 Grade efficiency under different inlet velocities

Apparently the grade efficiency is improved with the increase of liquid velocity. For smaller particles $(<0.18 \mathrm{~mm})$, the separation efficiency is $3.42,10.39$ and 20.88 percent corresponding to the velocity of $1.2 \mathrm{~m} / \mathrm{s}, 1.5 \mathrm{~m} / \mathrm{s}$ and $1.7 \mathrm{~m} / \mathrm{s}$, which is more significant than that of larger particles. Increasing inlet velocity, therefore, is an effective way in case small particles are required to be removed from liquid mixture.

\subsubsection{Oil/water ratio}

Actually, the oil/water ratio reflects the influence of mixture viscosity and mixture density on separation efficiency. In the experiments, the inlet velocity and particle size distribution keep unvaried for each run, 
while the water cut is $0.40,0.52,0.89$ and 1.0 respectively, and the experimental results are given in Fig. 6.

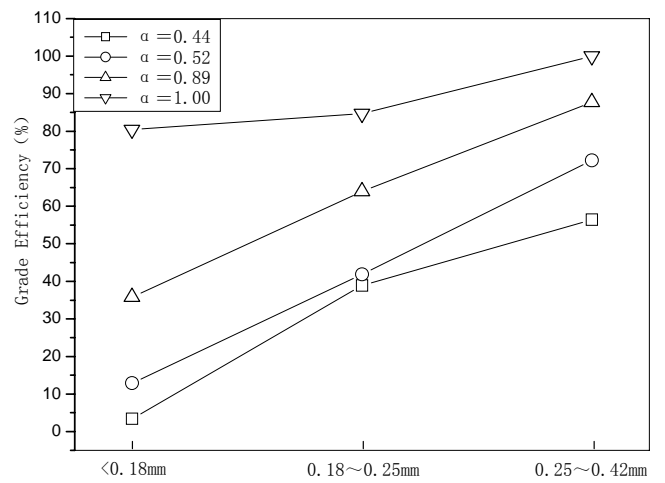

Fig. 6 Grade efficiency under different oil/water ratio

It indicates that the separation efficiency for all three grades enhances as the water cut increases. The separation efficiency of sand particles, especially those smaller ones, will drop dramatically when the water cut descends from 1.0 to 0.89 . Therefore, it is obvious that smaller particles $(<0.18 \mathrm{~mm})$ are more sensitive to the changes of water cut.

\subsubsection{Number of hydrocyclones}

In field applications, the separation efficiency is often improved by installing hydrocyclones in series. Thus, a new parameter $\eta_{21}$ is introduced here to investigate its influence on separation efficiency quantitatively, and the definition is:

$$
\eta_{21}=\frac{1}{1+\frac{m_{1}}{m_{2}}}
$$

where $m_{1}$ and $m_{2}$ are the mass of sand particles separated inside the first hydrocyclone and the second one respectively.

Fig. 7 shows the value of $\eta_{21}$ for different oil/ water ratio when the liquid velocity is $1.5 \mathrm{~m} / \mathrm{s}$, from which one can find that only a little sand is separated in the second hydrocyclone in case the liquid is pure water. However, as the water cut becomes $0.75,0.54$ and 0.42 , totally about $39.35,49.04$, and 70.46 percent of sand particles are separated in the second hydrocyclone, which even arrives at 56.53, 69.0 and 85.86 percent for smaller particles $(<0.18 \mathrm{~mm})$. Therefore, adding the number of hydrocyclones is effective to improve separation efficiency of sand particles (especially for smaller ones) in case of relatively high liquid viscosity.

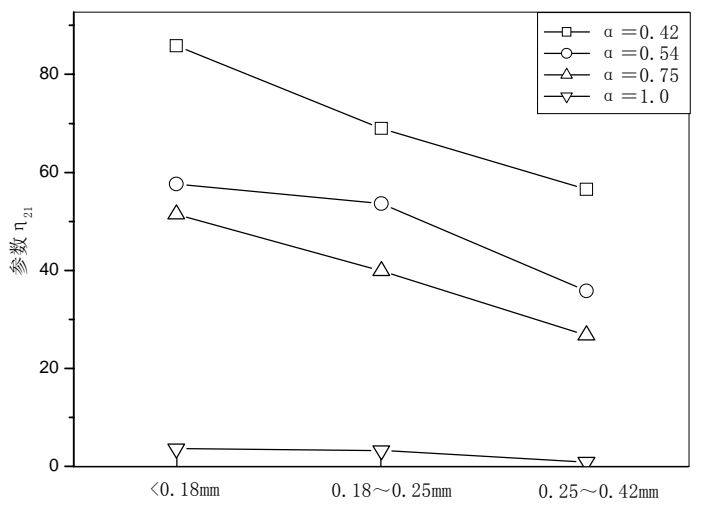

Fig. $7 \eta_{21}$ under different oil/water ratio

\subsubsection{Total efficiency}

Besides grade efficiency, total efficiency is the another important parameter in evaluating the performance of hydrocyclones. Three typical sets of experimental results are given in Table 1 .

As shown in Table 1, the total efficiency under different conditions are all higher than 95 percent, indicating that liquid/solid hydrocyclone, as a kind of compact, highly efficient separator, is very suitable to be applied to remove sand particles from liquid mixture on platforms.

Table 1 Some typical experimental results

\begin{tabular}{|c|c|c|c|c|c|c|}
\hline \multirow{2}{*}{$\begin{array}{c}\text { Inlet } \\
\text { velocity } \\
(\mathrm{m} / \mathrm{s})\end{array}$} & \multirow{2}{*}{$\begin{array}{c}\text { Water } \\
\text { cut } \\
(\%)\end{array}$} & \multicolumn{2}{|c|}{ Mass Distribution before Experiments $(\mathrm{g})$} & Mass of Separated & $\begin{array}{c}\text { Total } \\
\text { Particles }(\mathrm{g})\end{array}$ & $\begin{array}{c}\text { Efficiency } \\
(\%)\end{array}$ \\
\hline 1.7 & 41.7 & 1758.5 & 241.5 & 100 & 2058.1 & 98.0 \\
\hline 1.4 & 45.5 & 1320 & 1365 & 774 & 3360.5 & 97.2 \\
\hline 1.3 & 89.0 & 991 & 957 & 297 & 2184.0 & 97.3 \\
\hline
\end{tabular}

\section{Results and Analyses \\ 3.1 Theoretical analysis}

As can be seen from above, smaller particles are more sensitive to the changes of inlet velocities and oil/water ratio, and such a phenomenon can be explained theoretically as follows.

Neglecting the interactions among particles, forces on a particle in the radial direction mainly include centrifugal force $f_{c}$, buoyancy force $f_{b}$ and drag force $f_{d}$, and the expressions are as follows: 


$$
\begin{aligned}
& f_{c}=\frac{1}{2} \rho_{p} C \frac{\pi d_{p}^{2}}{4} v_{p l}^{2} \\
& f_{b}=\frac{1}{2} \rho_{l} C \frac{\pi d_{p}^{2}}{4} v_{p l}^{2} \\
& f_{d}=\frac{1}{2} \rho_{l} C_{D} \frac{\pi d_{p}^{2}}{4} v_{p l}^{2} \\
& C=\frac{4}{3} \frac{d_{p}}{r} v^{-2}, \bar{v}=\frac{v_{t}}{v_{p l}}
\end{aligned}
$$

where, $d_{p}$ - particle diameter, $[\mathrm{m}] ; r$ - motion gyration radius of a particle, $[\mathrm{m}] ; \rho_{p}$ - particle density, $\left[\mathrm{kg} / \mathrm{m}^{3}\right] ; \rho_{l}$ - liquid density, $\left[\mathrm{kg} / \mathrm{m}^{3}\right] ; \mu_{l}$ - liquid viscosity, $[\mathrm{Pa} \cdot \mathrm{s}] ; v_{t}$ - tangential velocity, $[\mathrm{m} / \mathrm{s}]$; $v_{p l}$ - relative velocity between particle and liquid, $[\mathrm{m}]$.

For spherical particles, the expression of $C_{D}$ within different ranges of the Reynolds number is ${ }^{[4]}$ :

$$
\begin{aligned}
& C_{D}=\frac{24}{\operatorname{Re}_{p l}}\left(1+0.15 \operatorname{Re}_{p l}^{0.687}\right), \operatorname{Re}_{p l} \leq 1000 ; \\
& C_{D}=0.44,1000<\operatorname{Re}_{p l} \leq 3 \times 10^{5} ; \\
& C_{D}=0.1, \quad \operatorname{Re}_{p l}>3 \times 10^{5} ;
\end{aligned}
$$

where the Reynolds number is defined as:

$$
\operatorname{Re}_{p l}=\frac{d_{p} v_{p l} \rho_{l}}{\mu_{l}}
$$

Usually the relative motion between particles and liquid is supposed to be laminar for small particles, especially inside small hydrocyclones, and in this case Eq. (7) is proposed to calculate the drag coefficient.

$$
C_{D}=\frac{24}{\operatorname{Re}_{p l}}
$$

A settling velocity $v_{p l o}$ is determined through balance of forces applied to a particle, expressed by

$$
v_{p l 0}=\frac{d_{p}^{2} v_{t}^{2}\left(\rho_{p}-\rho_{l}\right)}{18 \mu_{l} r}
$$

Obviously the higher the settling velocity, the more chances a particle would have to be separated. Because the settling velocity is directly proportional to the square of particle diameter, chances to be separated for smaller particles are more dependent on the inlet velocity and oil/water ratio, which increase as the inlet velocity or water cut becomes higher.

\subsection{Calculation of grade efficiency}

As described in the above, the separation efficiency of liquid/solid hydrocyclone depends on many parameters ${ }^{[b]}$, mainly including inlet velocity $v$, liquid viscosity $\mu_{l}$, liquid density $\rho_{l}$, solid concentration $c$, particle diameter $d$, solid density $\rho_{d}$, size distribution $G$, as well as geometrical dimensions of hydrocyclone ( such as hydrocyclone diameter $D$, height of hydrocyclone cylindrical part $L_{1}$, angle of hydrocyclone cone $\theta$, height of hydrocyclone cone $L_{2}$, vortex finder length $l$, overflow diameter $D_{o}$, underflow diameter $D_{u}$ and so on ), thus the separation efficiency $\eta$ can be expressed as

$$
\eta=f\left(d, \rho_{s}, \rho_{l}, \mu_{l}, v, c, G, D_{o}, D_{u}, L_{1}, L_{2}, \ldots\right)
$$

For a hydrocyclone with fixed dimensions, Eq. (10) can be obtained through dimensional analysis.

$$
\eta=f(S t k, \mathrm{Re})
$$

where,

$$
S t k=\left(\frac{\rho_{s}}{\rho_{l}}-1\right) \frac{\operatorname{Re}}{18 \bar{D}^{2}}, \quad \operatorname{Re}=\frac{\rho_{l} D v}{\mu_{l}}, \quad \bar{D}=\frac{D}{d}
$$

Therefore, the separation is a function of the Reynolds number and the Stokes number. Based on the experimental data, the curve of grade efficiency $\eta_{d}$ versus the Stokes number under different Reynolds numbers is given in Fig. 8, where the Reynolds number ranges from $1.0 \times 10^{4}$ to $2.5 \times 10^{4}$.

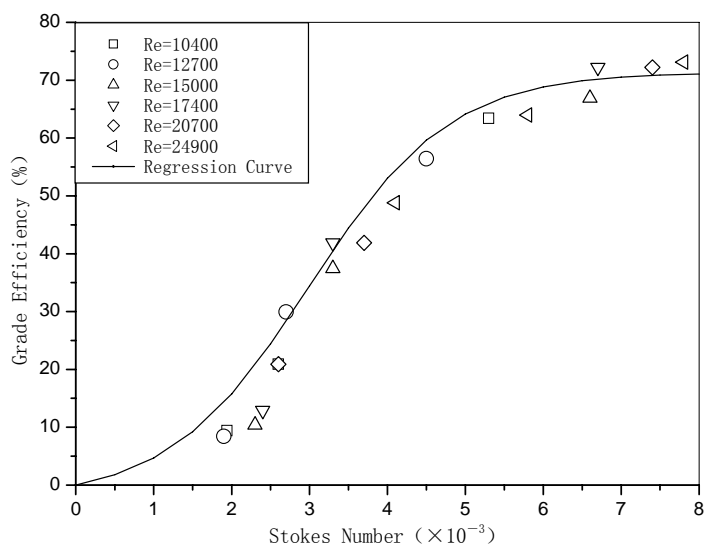

Fig. $8 \eta_{d}-$ Stk curve under different Reynolds numbers

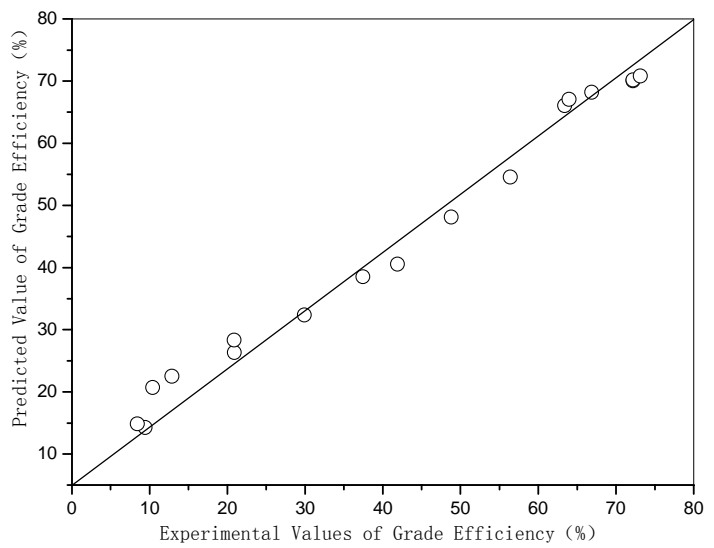

Fig. 9 Comparison of experimental and predicted values of grade efficiency 
It should be noted in Fig. 8 that data points under different Reynolds numbers present themselves in regular "S" shape distribution, which can be regressed using the Sigmoidal function, as

$$
\eta_{d}=71.38-\frac{73.93}{1+e^{[(1000 S t k-3) / 0.87]}}
$$

A comparison of experimental values and the calculated ones using Eq. (11) is shown in Fig. 9. Except for those points under low Stokes number, there is a reasonable fit between experimental values and the calculated ones.

\section{Conclusions}

Based on massive experiments, the relationship between separation efficiency and inlet velocity, oil/ water ratio, as well as the number of hydrocyclones is investigated, and the following conclusions can be drawn.

a) Liquid/solid hydrocyclone, as a kind of compact and highly efficient separator, is very suitable to be applied on oil platforms.

b) For a certain hydrocyclone, the separation efficiency mainly depends on inlet velocity, oil/water ratio and size distribution of particles.

c) Increasing inlet velocity or decreasing oil/water ratio can improve the separation efficiency of liquid/solid hydrocyclones.

d) Installing the second hydrocyclone is of little significance in case of low liquid viscosity.
However, as the oil/water ratio increases, more and more sand particles are separated at the second hydrocyclone.

e) Small particles are more sensitive to the changes of parameters, including inlet velocity, oil/water ratio and the number of hydrocyclones.

f) According to dimensional analysis, the separation efficiency is a function of the Reynolds number and Stokes number. When the Reynolds number varies between $1.0 \times 10^{4}$ and $2.5 \times 10^{4}$, there is a reasonable fit between experimental values and the calculated ones using regressed equation.

\section{References}

[1] Atakan Avci, Irfan Karagoz. Effects of flow and geometrical parameters on the collection efficiency in cyclone separators [J]. Aerosol Science, 2003, 34: 937 955.

[2] A. Avci, I. Karagoz. A mathematical model for the determination of a cyclone performance [J]. Int. Comm. Heat Mass Transfer, 2000, 27: 263 272

[3] A. C. Hoffmann, L. E. Stein. Gas Cyclones and Swirl Tubes [M]. Springer, 2002

[4] C. R. Parker. Aerosol Science and Technology [M]. New York: McGraw-Hill Inc, 1993.

[5] M. A. Z. Coelho, R. A. Medronho. A model for performance prediction of hydrocyclones [J]. Chemical Engineering Journal, 2001, 84: 7 14

[6] L. Svarovsky, M. T. Thew. Hydrocyclones - Analysis and Applications. Kluwer Academic Publishers, 1992. 